\title{
INVARIANT MEANS ON LOCALLY COMPACT SEMIGROUPS
}

\author{
JAMES C. S. WONG ${ }^{1}$
}

\begin{abstract}
Let $G$ be a locally compact semigroup (jointly continuous semigroup operation), $M(G)$ the algebra of all bounded regular Borel measures on $G$ (with convolution as multiplication), $E$ a separated locally convex space and $S$ a compact convex subset of $E$. We show that there is a left invariant mean on the space LUC $(G)$ of all bounded left uniformly continuous functions on $G$ iff $G$ has the following fixed point property: For any bilinear mapping $T: M(G) \times E \rightarrow E$ (denoted by $(\mu, s) \rightarrow T_{\mu}(s)$ ) such that (a) $T_{\mu}(S) \subset S$ for any $\mu \geqq 0,\|\mu\|=1$, (b) $T_{\mu * \nu}=T_{\mu} \circ T_{\nu}$ for any $\mu$, $\nu \in M(G)$, (c) $T_{\mu}: S \rightarrow S$ is continuous for any $\mu \geqq 0,\|\mu\|=1$, and (d) $\mu \rightarrow T_{\mu}(s)$ is continuous for each $s \in S$ when $M(G)$ has the topology induced by the seminorms $p_{f}(\mu)=\left|\int f d \mu\right|, f \in \operatorname{LUC}(G)$, there is some $s_{0} \in S$ such that $T_{\mu}\left(s_{0}\right)=s_{0}$ for any $\mu \geqq 0,\|\mu\|=1$.
\end{abstract}

1. Introduction. The purpose of this paper is to extend certain results in the theory of invariant means on locally compact groups to locally compact semigroups. Let $G$ be a locally compact group with measure algebra $M(G)$. The present author has proved in [17, Theorem 3.3] that $L_{\infty}(G)^{2}$ has a topological left invariant mean iff $G$ has the fixed point property on convex compacta for separately continuous actions of $M(G)$. In this paper, we shall prove among other things, that if $G$ is a locally compact semigroup, then there is a left invariant mean on the space of all bounded left uniformly continuous functions on $G$ iff $G$ has a similar fixed point property which turns out to be equivalent to the above fixed point property when $G$ is a locally compact group.

\section{Terminologies.}

2.1 The measure algebra $M(G)$. A locally compact semigroup is a semigroup with a locally compact topology for which the semigroup

Received by the editors February 25, 1970 and, in revised form, October 26, 1970.

AMS 1968 subject classifications. Primary 2090, 2205, 4256.

Key words and phrases. Locally compact semigroups, fixed point properties, invariant means, convolution algebras, left uniformly continuous functions.

${ }^{1}$ This research was supported by a National Research Council of Canada Postdoctoral Fellowship at McMaster University.

${ }^{2} L_{\infty}(G)$ is defined with respect to a fixed left Haar measure (see Hewitt and Ross [9, Definition 12.11]). For definition of topological left invariant mean, consult [8] or [16].

(c)American Mathematical Society 1972 
operation is jointly continuous. Let $G$ be a locally compact semigroup and $M(G)$ the Banach space of all bounded regular Borel measures on $G$ [9, Definition 14.9]. It is known that $M(G)$ is a convolution algebra if we define the convolution $\mu * v$ of two measures $\mu, v$ in $M(G)$ by the formula

$$
\int f(z) d(\mu * v)(z)=\iint f(x y) d \mu(x) d v(y)
$$

for any $f \in C_{0}(G)$, the continuous functions on $G$ which vanish at infinity (see Glicksberg [6]). It follows immediately that the same formula is valid for any $f \in L_{1}(G,|\mu| *|\nu|)(|\mu|$ denotes the total variation of $\mu$; see Hewitt and Ross [9, Theorems 14.6 and 14.14]). This can be proved by repeating "mutatis mutandis" the arguments used in Hewitt and Ross [9, Theorem 19.10], where $G$ is assumed to be a locally compact group (because only the continuity of the map $(x, y) \rightarrow x y$ of $G \times G$ into $G$ is invoked in their proof). Let $M_{0}(G)$ be the set of all probability measures in $M(G)\left(\mu \in M_{0}(G)\right.$ iff $\mu \geqq 0$ and $\left.\|\mu\|=1\right)$. It is easy to see that $M_{0}(G)$ is a convolution semigroup.

2.2 The space $\operatorname{LUC}(G)$ and its dual. Let $\mathrm{CB}(G)$ be the Banach space of all continuous bounded functions on the locally compact semigroup $G$. A function $f \in \operatorname{CB}(G)$ is called left uniformly continuous ${ }^{3}$ if the map $a \rightarrow l_{a} f$ of $G$ into $\mathrm{CB}(G)$ is norm continuous $\left(l_{a} f(x)=f(a x), x \in G\right)$. The space of all left uniformly continuous functions on $G$ is denoted by $\operatorname{LUC}(G)$. It is known that $\operatorname{LUC}(G)$ is a translation invariant linear subspace of $\operatorname{CB}(G)$ containing the constants and is left introverted in the sense that if $f \in \operatorname{LUC}(G), m \in \operatorname{LUC}(G)^{*}$, then $m_{l}(f) \in \operatorname{LUC}(G)$ where $m_{l}(f)$ is defined by $m_{l}(f)(x)=m\left(l_{x} f\right), x \in G$. (See Namioka [12] or Mitchell [11].) The space $\operatorname{LUC}(G) *$ can be made into a Banach algebra if we define the Arens product $m \odot n$ of two functionals $m, n$ in $\operatorname{LUC}(G)^{*}$ by $m \odot n(f)=m\left(n_{l}(f)\right)$ for any $f \in \operatorname{LUC}(G)$.

For each $f \in \operatorname{LUC}(G)$, define a seminorm [14, Chapter I, $\S 4] p_{f}$ on the linear space $M(G)$ by $p_{f}(\mu)=\left|\int f d \mu\right|, \mu \in M(G)$. The locally convex topology on $M(G)$ determined by these seminorms [14, Theorem 3, p. 15] is denoted by $\tau$. Note that each $\mu \in M(G)$ can be regarded as a functional in $\operatorname{LUC}(G)^{*}$ if we set $\bar{\mu}(f)=\int f d \mu, f \in \operatorname{LUC}(G)$. (But this embedding might not be one to one, in other words, $\tau$ might not be separated. If $G$ is a locally compact group, then $C_{0}(G) \subset \operatorname{LUC}(G)$. Hence $\tau$ is separated and therefore the same as the $w^{*}$ topology of $\operatorname{LUC}(G)^{*}$ restricted to $M(G)$.)

A functional $m$ in $\operatorname{LUC}(G)^{*}$ is called a mean iff $\|m\|=m(1)=1$. A mean $m$ is left invariant if $m\left(l_{a} f\right)=m(f)$ for any $a \in G, f \in \operatorname{LUC}(G)$.

\footnotetext{
${ }^{3}$ Some authors call these functions right uniformly continuous and denote them by $\mathrm{UCB}_{r}(G)$ (see for example Greenleaf [8]).
} 
3. A lemma. We shall need the following lemma which gives some useful information about the spaces $M(G), \operatorname{LUC}(G)$ and its dual LUC $(G)^{*}$. It is also of independent interest.

LEMMA 3.1. Let $G$ be a locally compact semigroup, then:

(a) For each $\mu$ in $M(G)$, the map $m \rightarrow \bar{\mu} \odot m$ is $w^{*}-w^{*}$ continuous on any norm bounded subset of $\mathrm{LUC}(G)^{*}$.

(b) For each $m$ in $\operatorname{LUC}(G)^{*}$, the map $\mu \rightarrow \bar{\mu} \odot m$ is continuous with respect to the topology $\tau$ of $M(G)$ and the $w^{*}$ topology of $\mathrm{LUC}(G) *$.

(c) If $\mu, v \in M(G)$, then $\bar{\mu} \odot \bar{\nu}=\overline{\mu * v}$ on $\operatorname{LUC}(G)$.

(d) $A$ mean $m$ in $\operatorname{LUC}(G)^{*}$ is left invariant iff $\bar{\mu} \odot m=m$ for any $\mu$ in $M_{0}(G)$.

(e) $\operatorname{LUC}(G)$ has a left invariant mean iff there is a net $\mu_{\alpha}$ in $M_{0}(G)$ such that $\mu * \mu_{\alpha}-\mu_{\alpha} \stackrel{\tau}{\rightarrow} 0$ in $M(G)$ for any $\mu$ in $M_{0}(G)$.

Proof. (a) Let $m_{\alpha} \rightarrow m$ in weak* topology of $\operatorname{LUC}(G)^{*}$ and $\left\|m_{\alpha}\right\|$, $\|m\| \leqq K$. For each $f$ in $\operatorname{LUC}(G), s, t \in G$,

$$
\left|\left(m_{\alpha}\right)_{l} f(s)-\left(m_{\alpha}\right)_{l} f(t)\right|=\left|m_{\alpha}\left(l_{s} f\right)-m_{\alpha}\left(l_{t} f\right)\right| \leqq K \cdot\left\|l_{s} f-l_{t} f\right\| .
$$

Therefore the family of functions $\left(m_{\alpha}\right)_{l} f$ is equicontinuous (Kelley $\left[10\right.$, p. 232]). Since $\left(m_{\alpha}\right)_{l} f \rightarrow m_{l}(f)$ pointwise on $G$, the convergence is uniform on every compact subset of $G$ (Kelley [10, Theorem 7.15]). Let $\mu$ in $M(G)$ have compact support, then $\bar{\mu} \odot m_{\alpha}(f)-\bar{\mu} \odot m(f)=$ $\int\left(m_{\alpha}\right)_{l} f d \mu-\int m_{l}(f) d \mu \rightarrow 0$.

Since the measures in $M(G)$ with compact supports are norm dense in $M(G)$ and $\left\|\left(m_{\alpha}\right)_{l} f\right\| \leqq K \cdot\|f\|$, it follows that $\bar{\mu} \odot m_{\alpha} \rightarrow \bar{\mu} \odot m$ in $w^{*}$ topology of $\operatorname{LUC}(G)^{*}$, for any $\mu$ in $M(G)$.

(b) Let $m$ in LUC $(G)^{*}$ be fixed. Suppose $\mu_{\alpha} \rightarrow \mu$ in the topology $\tau$ of $M(G)$, then $\bar{\mu}_{\alpha} \odot m(f)-\bar{\mu} \odot m(f)=\int m_{l}(f) d \mu_{\alpha}-\int m_{l}(f) d \mu \rightarrow 0$ since $m_{l}(f) \in \operatorname{LUC}(G)$ for any $f \in \operatorname{LUC}(G)$. Therefore $\bar{\mu}_{\alpha} \odot m \rightarrow \bar{\mu} \odot m$ in weak* topology of $\operatorname{LUC}(G)^{*}$.

(c) Let $\mu, v \in M(G)$, then

$$
\begin{aligned}
\bar{\mu} \odot \bar{\nu}(f) & =\bar{\mu}\left(\bar{v}_{l}(f)\right)=\int \bar{v}_{l}(f)(x) d \mu(x)=\int \bar{\nu}\left(l_{x} f\right) d \mu(x) \\
& =\iint f(x y) d \mu(x) d v(y)=\int f(z) d(\mu * v)(z)=\overline{\mu * v}(f),
\end{aligned}
$$

$f \in \operatorname{LUC}(G)$. (Recall the remarks in §2.1.) Hence

on $\operatorname{LUC}(G)$.

$$
\bar{\mu} \odot \bar{\nu}=\overline{\mu * v}
$$

(d) If $m \in \operatorname{LUC}(G)^{*}$ is such that $\bar{\mu} \odot m=m$ for any $\mu \in M_{0}(G)$, then clearly $m$ is left invariant since $M_{0}(G)$ contains the point measures. But the 
convex combinations of these point measures are $w^{*}$ dense in the set of means in $\operatorname{LUC}(G)^{*}$ (more precisely, it is their images under the map $\mu \rightarrow \bar{\mu}$ which are $w^{*}$ dense). Therefore if $m$ is left invariant and $\mu \in$ $M_{0}(G)$, let $\mu_{\alpha}$ be a net of convex combinations of point measures such that $\bar{\mu}_{\alpha} \stackrel{w^{*}}{\rightarrow} \bar{\mu}$ in $\operatorname{LUC}(G)^{*}$, then $\mu_{\alpha} \stackrel{\tau}{\rightarrow} \mu$ in $M(G)$ and hence $\bar{\mu}_{\alpha} \odot m \stackrel{w^{*}}{\rightarrow}$ $\bar{\mu} \odot m$ in $\operatorname{LUC}(G)^{*}$ by $(b)$. Now clearly $\bar{\mu}_{\alpha} \odot m=m$ since $m$ is left invariant. Consequently $\bar{\mu} \odot m=m$.

(e) Suppose $\operatorname{LUC}(G)$ has a left invariant mean $m$, then by (d), $\bar{\mu} \odot m=m$ for any $\mu \in M_{0}(G)$ which is $w^{*}$ dense in the set of means in $\operatorname{LUC}(G)^{*}$. Let $\mu_{\alpha} \in M_{0}(G)$ be a net such that $\bar{\mu}_{\alpha} \stackrel{w^{*}}{\rightarrow} m$ in $\operatorname{LUC}(G)^{*}$. Since $\left\|\bar{\mu}_{\alpha}\right\| \leqq\left\|\mu_{\alpha}\right\|=1$ and $\|m\|=1$, by $(\dot{\mathrm{a}}), \vec{\mu} \odot \bar{\mu}_{\alpha} \stackrel{w^{*}}{\rightarrow} \bar{\mu} \odot m$ in $\operatorname{LUC}(G)^{*}$. Therefore

$$
\overline{\mu * \mu_{\alpha}-\mu_{\alpha}}=\overline{\mu * \mu_{\alpha}}-\bar{\mu}_{\alpha}=\bar{\mu} \odot \bar{\mu}_{\alpha}-\bar{\mu}_{\alpha} \stackrel{w^{*}}{\rightarrow} \bar{\mu} \odot m-m=0
$$

in $\operatorname{LUC}(G) *$ by (c). In other words $\mu * \mu_{\alpha}-\mu_{\alpha} \stackrel{\tau}{\rightarrow} 0$ in $M(G)$.

Conversely assume that for some net $\mu_{\alpha}$ in $\vec{M}_{0}(G), \mu * \mu_{\alpha}-\mu_{\alpha} \stackrel{\tau}{\rightarrow} 0$ for any $\mu \in M_{0}(G)$. By $w^{*}$ compactness of the set of means in $\operatorname{LUC}(G)^{*}$, we can assume $\bar{\mu}_{\alpha} \stackrel{w^{*}}{\rightarrow} m$ for some mean $m$ in $\operatorname{LUC}(G)^{*}$ (passing to a subnet if necessary). Then

$$
\begin{aligned}
\bar{\mu} \odot m-m & =\bar{\mu} \odot\left(w^{*} \lim _{\alpha} \bar{\mu}_{\alpha}\right)-w^{*} \lim _{\alpha} \cdot \bar{\mu}_{\alpha} \\
& =w^{*} \lim _{\alpha}\left(\bar{\mu} \odot \bar{\mu}_{\alpha}-\bar{\mu}_{\alpha}\right)=w^{*} \lim _{\alpha}\left(\overline{\mu * \mu_{\alpha}-\mu_{\alpha}}\right)=0
\end{aligned}
$$

by (a) and (c). Hence $m$ is a left invariant mean on $\operatorname{LUC}(G)$.

\section{Main theorems.}

DEFINITION 4.1. Let $G$ be a locally compact semigroup, $E$ a separated locally convex space. An action $T$ of $M(G)$ on $E$ is a homomorphism of $M(G)$ into the algebra of linear operators in $E$. Thus we have a bilinear map $T: M(G) \times E \rightarrow E$ (where $(\mu, s) \rightarrow T_{\mu}(s), \mu \in M(G), s \in E$ ) such that $T_{\mu * \nu}=T_{\mu} \circ T_{\nu}$ for any $\mu, \nu \in M(G)$. If $S$ is a compact convex subset of $E$, we say that $S$ is $M_{0}(G)$-invariant under $T$ if $T_{\mu}(S) \subset S$ for any $\mu \in$ $M_{0}(G)$. In this case $T$ induces an action $T: M_{0}(G) \times S \rightarrow S$ of the convolution semigroup $M_{0}(G)$ on $S$ as affine maps in $S$ (see [17] for definition of actions in the case when $G$ is a locally compact group).

THEOREM 4.2. Let $G$ be a locally compact semigroup, then the following conditions are equivalent:

(a) $\mathrm{LUC}(G)$ has a left invariant mean.

(b) If $T: M(G) \times E \rightarrow E$ is any action of $M(G)$ on a separated locally convex space $E$ and $S$ any compact convex $M_{0}(G)$-invariant subset of $E$ such that (i) for each $\mu \in M_{0}(G), T_{\mu}: S \rightarrow S$ is continuous and (ii) for each 
$s \in S$, the map $\mu \rightarrow T_{\mu}(s)$ from $M(G)$ into $E$ is continuous when $M(G)$ has the topology $\tau$, then the induced action $T: M_{0}(G) \times S \rightarrow S$ has a fixed point.

Proof. Assume that $\operatorname{LUC}(G)$ has a left invariant mean. By Lemma 3.1(e), there is a net $\mu_{\alpha} \in M_{0}(G)$ such that $\mu * \mu_{\alpha}-\mu_{\alpha} \stackrel{\tau}{\rightarrow} 0$ in $M(G)$. Let $T: M(G) \times E \rightarrow E$ be any action of $M(G)$ on $E$ and $S \subset E$ a compact convex $M_{0}(G)$-invariant subset of $E$ satisfying conditions (i) and (ii) of (b). Consider the net $T_{\mu_{\alpha}}(s)$ in $S$ where $s \in S$ is arbitrary but fixed. By compactness of $S$, we can assume $T_{\mu_{\alpha}}(s) \rightarrow s_{0}$ in $S$ (use a subnet if necessary). We shall show that $s_{0}$ is the required fixed point of the action $T: M_{0}(G) \times$ $S \rightarrow S$ by repeating the arguments used in the proof of [17, Theorem 3.1]. Let $\mu \in M_{0}(G)$, then

$$
\begin{aligned}
T_{\mu}\left(s_{0}\right) & =T_{\mu}\left(\lim _{\alpha} T_{\mu_{\alpha}}(s)\right)=\lim _{\alpha} T_{\mu}\left(T_{\mu_{\alpha}}(s)\right)=\lim _{\alpha} T_{\mu \star \mu_{\alpha}}(s) \\
& =\lim _{\alpha}\left\{T_{\mu * \mu_{\alpha}-\mu_{\alpha}}(s)+T_{\mu_{\alpha}}(s)\right\}=\lim _{\alpha} T_{\mu_{\alpha}}(s)=s_{0}
\end{aligned}
$$

by condition (i), linearity of $\mu \rightarrow T_{\mu}(s)$, condition (ii) and the fact that $\mu * \mu_{\alpha}-\mu_{\alpha} \stackrel{\tau}{\rightarrow} 0$ in $M(G)$.

Conversely, assume (b). Let $E=\operatorname{LUC}(G)^{*}$ with $w^{*}$ topology and $S=$ the set of means in $\operatorname{LUC}(G)^{*}$. Define an action of $M(G)$ on $E$ by $T_{\mu}(m)=\bar{\mu} \odot m$ for each $\mu \in M(G), m \in \operatorname{LUC}(G)^{*}$. It is clear that the map $T: M(G) \times E \rightarrow E$ is bilinear. To show that $T$ is an action, suppose $\mu, v \in M(G)$, then

$$
T_{\mu * \nu}(m)=\overline{\mu * \nu} \odot m=(\bar{\mu} \odot \bar{\nu}) \odot m=\bar{\mu} \odot(\bar{\nu} \odot m)=T_{\mu}\left(T_{\nu}(m)\right)
$$

by Lemma 3.1(c) and associativity of the Arens product. Hence $T_{\mu * \nu}=$ $T_{\mu} \circ T_{v}$. Obviously $S$ is a $w^{*}$ compact convex subset of $\operatorname{LUC}(G)^{*}$. If $m \in S, \quad \mu \in M_{0}(G)$ then $\left\|T_{\mu}(m)\right\| \leqq\|\mu\| \cdot\|m\|=1$ and $T_{\mu}(m)(1)=$ $(\bar{\mu} \odot m)(1)=1$. Consequently $\left\|T_{\mu}(m)\right\|=T_{\mu}(m)(1)=1$ and $T_{\mu}(m)$ is a mean on $\operatorname{LUC}(G)$. Thus $S$ is $M_{0}(G)$-invariant under $T$. By Lemma 3.1(a) and (b), it is straightforward to verify that the action $T$ defined above satisfies the continuity conditions (i) and (ii) of (b). Therefore by assumption (b), the induced action $T: M_{0}(G) \times S \rightarrow S$ must have a fixed point which is a left invariant mean on $\mathrm{LUC}(G)$ by Lemma 3.1(d). This completes the proof of the theorem.

REMARK 4.3. Suppose $T: M(G) \times E \rightarrow E$ is any action of $M(G)$ on $E$ and $S$ a compact convex $M_{0}(G)$-invariant subset of $E$ satisfying the continuity conditions (i) and (ii) of (b) in the theorem. Let $T: G \times S \rightarrow S$ be defined by $T_{x}(s)=T_{\mu(x)}(s)$ where $x \in G, s \in S$ and $\mu(x)$ is the point measure at the point $x . T$ is an action of $G$ as affine maps in $S$ (that is, each map $T_{x}: S \rightarrow S$ is affine and $T_{x y}=T_{x} \circ T_{y}$ for any $\left.x, y \in G\right)$. Moreover, the map $(x, s) \rightarrow T_{x}(s)$ is separately continuous. For if $x_{\alpha} \rightarrow x$ in 
$G$, then $\mu\left(x_{\alpha}\right) \stackrel{\tau}{\rightarrow} \mu(x)$ in $M(G)$ and hence $T_{x_{\alpha}}(s)=T_{\mu(x)_{\alpha}}(s) \rightarrow T_{\mu(x)}(s)=$ $T_{x}(s)$ in $S$ by (ii) while continuity of the map $s \rightarrow T_{x}(s)$ follows from (i). In general, this is all we can say about the action $T: G \times S \rightarrow S$ of $G$. However if $G$ is a locally compact group, then the same action, being separately continuous, is also jointly continuous by a theorem of Ellis [5, Theorem 1]. Consequently, if we assume that $\operatorname{LUC}(G)$ has a left invariant mean, then the action $T: G \times S \rightarrow S$ must have a fixed point by Rickert's theorem [13, Theorem 4.2]; see also Mitchell [11, Theorem 2] for a more general result. It follows that $s_{0}$ is also a fixed point of the induced action $T: M_{0}(G) \times S \rightarrow S$ (because the convex combinations of point measures are $w^{*}$ dense in the set of means in $\left.\operatorname{LUC}(G)^{*}\right)$. This gives yet another proof of (a) implies (b) in the case when $G$ is a locally compact group.

\section{Special cases.}

5.1 Locally compact group. Let $G$ be a locally compact group. It was proved in [17] that $L_{\infty}(G)$ has a topological left invariant mean (see [16] for definition) iff $G$ has the following fixed point property:

(*) For any action $T: M(G) \times E \rightarrow E$ of $M(G)$ on a separated locally convex space $E$ and any compact convex $M_{0}(G)$-invariant subset $S$ of $E$ such that the map $M(G) \times E \rightarrow E$ is separately continuous when $M(G)$ has the norm topology, the induced action $T: M_{0}(G) \times S \rightarrow S$ has a fixed point.

Now it is known that $L_{\infty}(G)$ has a topological left invariant mean iff $\operatorname{LUC}(G)$ has a left invariant mean (see Greenleaf [8] where $\operatorname{LUC}(G)$ is denoted by $\mathrm{UCB}_{r}(G)$ ). Therefore we have the following theorem.

THEOREM 5.2. Let $G$ be a locally compact group, then the following are equivalent:

(a) $L_{\infty}(G)$ has a topological left invariant mean.

(b) LUC(G) has a left invariant mean.

(c) $G$ has fixed point property (b) of Theorem 4.2.

(d) $G$ has fixed point property (*) of 5.1 .

5.3 Compact semigroups. Suppose that $G$ is a compact semigroup. It is well known that $\mathrm{CB}(G)=\operatorname{LUC}(G)$ (Namioka [12]) and that $\mathrm{CB}(G)$ has a left invariant mean iff the kernel $K(G)$ of $G$ is a compact group and the unique invariant mean is the Haar integral over $K(G)$ (see Rosen [15] or Glicksberg and de Leeuw [7]). Let $v$ be the normalised Haar measure of $K(G)$, define $\lambda \in M_{0}(G)$ by $\int f d \lambda=\left.\int_{K(G)} f\right|_{K(G)} d \nu, f \in C_{0}(G)=\mathrm{CB}(G)$. By direct calculation, one shows that $\int f d \mu * \lambda=\int f d \lambda$ for any $\mu \in M_{0}(G)$ and $f \in C_{0}(G)$. Hence $\mu * \lambda=\lambda$. Now if $T: M(G) \times E \rightarrow E$ is any action of $M(G)$ on $E$ and $S \subset E$ is compact convex and $M_{0}(G)$-invariant, then $T_{\mu}\left(T_{\lambda}(s)\right)=T_{\mu * \lambda}(s)=T_{\lambda}(s)$ for any $\mu$ in $M_{0}(G)$ and $s \in S$. In other words, $T_{\lambda}(s)$ is a fixed point of the induced action $T: M_{0}(G) \times S \rightarrow S$, for each 
$s \in S$, without any continuity conditions on the action $T: M(G) \times E \rightarrow E$ whatsoever.

Finally, it is also interesting to note that when $G$ is compact, the mapping $\mu \rightarrow \bar{\mu}$ is precisely the natural isometric isomorphism $M(G)=$ $C_{0}(G)^{*}$, and the Arens product $\odot$ is nothing but convolution of measures in $M(G)$.

\section{REFERENCES}

1. M. M. Day, Amenable semigroups, Illinois J. Math. 1 (1957), 509-544. MR 19, 1067.

2. - Fixed-point theorems for compact convex sets, Illinois J. Math. 5 (1961), 585-590. MR 25 \#1547.

3. — Correction to my paper, "Fixed-point theorems for compact convex sets," Illinois J. Math. 8 (1964), 713. MR 29 \#6463.

4. N. Dunford and J. T. Schwartz, Linear operators. I: General theory, Pure and Appl. Math., Vol. 7, Interscience, New York, 1958. MR 22 \#8302.

5. R. Ellis, Locally compact transformation groups, Duke Math. J. 24 (1957), 119-125. MR 19, 561.

6. I. Glicksberg, Weak compactness and separate continuity, Pacific J. Math. 11 (1961), 205-214. MR 22 \#11275.

7. K. de Leeuw and I. Glicksberg, Applications of almost periodic compactifications, Acta Math. 105 (1961), 63-97. MR 24 \#A1632.

8. F. P. Greenleaf, Invariant means on topological groups and their applications, Van Nostrand Math Studies, no. 16, Van Nostrand, Princeton, N.J., 1969. MR 40 \#4776.

9. E. Hewitt and K. A. Ross, Abstract harmonic analysis. Vol. 1: Structure of topological groups. Integration theory, group representations, Die Grundlehren der math. Wissenschaften, Band 115, Academic Press, New York; Springer-Verlag, Berlin, 1963. MR 28 \#158.

10. J. Kelley, General topology, Van Nostrand, Princeton, N.J., 1955. MR 16, 1136.

11. T. Mitchell, Topological semigroups and fixed points, Illinois J. Math. 14 (1970), 630-641.

12. I. Namioka, On certain actions of semigroups on L-spaces, Studia Math. 29 (1967), 63-77. MR 36 \#6910.

13. N. Rickert, Amenable groups and groups with the fixed point property, Trans. Amer. Math. Soc. 127 (1967), 211-232. MR 36 \#5260.

14. A. P. Robertson and W. J. Robertson, Topological vector spaces, Cambridge Univ. Press, New York, 1964. MR 28 \#5318.

15. W. G. Rosen, On invariant means over compact semigroups, Proc. Amer. Math. Soc. 7 (1956), 1076-1082. MR 18, 495.

16. James C. S. Wong, Topologically stationary locally compact groups and amenability, Trans. Amer. Math. Soc. 144 (1969), 351-363. MR 40 \#2781.

17. - Topological invariant means on locally compact groups and fixed points, Proc. Amer. Math. Soc. 27 (1971), 572-578.

Department of Mathematics, McMaster University, Hamilton, Ontario, CANADA

Current address: Department of Mathematics, University of Calgary, Calgary 44, Alberta, Canada 